\title{
NONDESTRUCTIVE CONTROL OF CONDITION AND PREDICTION OF CABLE LIFE TIME AT NUCLEAR POWER PLANTS
}

\author{
Kononenko A.I. \\ Research Institute of \\ Scientific Instruments \\ kononenko@niipriborov.ru
}

\author{
Chlenov A.M. \\ Research Institute of \\ Scientific Instruments \\ chlenov@niipribor.ru
}

\author{
Tsikanin A.G. \\ Research Institute of \\ Scientific Instruments \\ tsikanin@niipriborov.ru
}

\section{INTRODUCTION}

The most effective methods for nondestructive control of a cable condition, which are used for a periodic assessment of expected life of power and control cables at nuclear power plants, are presented. In spite of the fact that many methods are based on physical principles that are well-known for a long time, they have been improved due to the experimental data received lately.

\section{EFFECTIVE METHODS OF NONDESTRUCTIVE CABLE MONITORING}

The conservative estimate of polyolefin insulation material life is realized on the basis of two tests of microsamples in a cell of the DSC device: isothermal mode for measuring oxidation time (OIT) and mode of temperature ramp for measuring oxidation temperature (OITP) [1].

Resulting DSC analysis of insulation with the different aging degrees allowed to receive equations for the expected life XLPE cables at different operating conditions, particularly, under conditions of simultaneous exposure to $\gamma$-radiation and elevated temperature, it is possible to use the equation:

$$
\tau_{E L_{-} T R}(T, \gamma)=a \cdot \exp \left(\frac{E_{a}}{R T}\right) \cdot \exp (-k \cdot D),
$$

where $E_{a}$ - the activation energy of antioxidant oxidation process, $a$ - the constant preexponential multiplier, $R$ - the Boltzmann constant, $T$ - the operation temperature, $D$ the total dose, $k$ - is the factor depending on a type of insulation.

Application of the IR Fourier spectroscopy with an attachment of the broken total internal reflection for work with microsamples gives possibility to define a type of polymeric insulation and to estimate a degree of its ageing on characteristic band of absorption spectra. For example, the peak optical density at a wave length of $1720 \mathrm{~cm}^{-1}$ is determined by a plasticizer concentration in PVC insulation; taking into consideration the exponential dependence of plasticizer concentration on time of ageing, the expected life for PVC cables is determined by the equation:

$$
\tau_{E L_{-} C}=\tau_{\text {test }} \cdot \frac{\ln C_{i n i}-\ln C_{\text {lim }}}{\ln C_{i n i}-\ln C_{\text {test }}},
$$

where $C_{\text {ini }}, C_{\text {lim }}, C_{\text {test }}$ are the initial, limiting content and measured values of plasticizer, respectively.

Cable polymeric Indenter is traditionally applied to estimating a degree of I\&C cable ageing using value of the compression module [1]. For the expected life estimation by the compression module the following equation is used:

$$
\tau_{E L_{-} M}=\tau_{\text {test }} \cdot \frac{M_{\text {lim }}-M_{\text {test }}}{M_{\text {test }}-M_{\text {ini }}},
$$

where $M_{\text {ini }}, M_{\text {lim }}, M_{\text {test }}$ are the initial, limiting, and measured values of the compression module, respectively.

The new approach [2] to the estimation of a technical condition of power cable electric insulation on the basis of the recovered voltage measurement allows monitoring a change in a bulk charge condition and electrical conductivity of insulation by results of one measurement.

For power cables with the impregnated paper insulation, which are laid out of containment in old power units of the nuclear power plants, this approach enables to monitor such typical defects under operation as drying out of the impregnant and insulation humidifying across all the way of its ageing from faultless to limiting conditions.

\section{CONCLUSIONS}

The presented nondestructive condition monitoring methods for I\&C cables and power cables with the impregnated paper insulation give the opportunity to make a periodic estimation of their expected life without destruction of cable lines.

\section{REFERENCES}

[1] IAEA-TECDOC-1188, Volume 1-2, ISSN 1011-4289. Vienna: IAEA, 2000.

[2] Kononenko A.I., Hohryakov A.V., "Estimation of a paper electric insulation condition by the results of measurements of the recovered voltage", Electrical Engineering, S.47-55, No.5, 2010. 


\section{NONDESTRUCTIVE CONTROL OF CONDITION AND PREDICTION OF CABLE LIFE TIME AT NUCLEAR POWER PLANTS}

\author{
Kononenko A.I. \\ Department of \\ Environmental \\ Qualification \\ Research Institute of \\ Scientific Instruments \\ Lytkarino, Moscow region \\ 140080 \\ Russia \\ Phone: +7 4955523807 \\ kononenko@niipriborov.ru
}

\author{
Chlenov A.M. \\ Director \\ Research Institute of \\ Scientific Instruments \\ Lytkarino, Moscow region \\ 140080 \\ Russia \\ Phone: +7 4955523931 \\ chlenov@niipribor.ru
}

\author{
Tsikanin A.G. \\ Department of \\ Environmental \\ Qualification \\ Research Institute of \\ Scientific Instruments \\ Lytkarino, Moscow region \\ 140080 \\ Russia \\ Phone: +7 4955523807 \\ tsikanin@niipriborov.ru
}

Keywords: Cables, diagnostics, differential scanning calorimetry, infrared spectroscopy, Indenter measurements, recovered voltage.

\begin{abstract}
The most effective methods for nondestructive control of a cable condition, which are used for a periodic assessment of expected life of power and control cables at nuclear power plants of Rosenergoatom Concern OJSC, are presented. In spite of the fact that many methods are based on physical principles that are well-known for a long time, they have been improved due to the experimental data received lately.
\end{abstract}

\section{INTRODUCTION}

Methods for monitoring and diagnostics of low-voltage I\&C cables, as a rule, are based on measurement of physical and chemical properties of insulation materials which allow fixing and monitoring a degree of their ageing at an early stage up to a limiting condition [1, 2]. Thus the condition monitoring is carried out in "hot spots" of cable lines. Such monitoring methods as a differential scanning calorimetry (DSC), IR Fourier spectroscopy of microsamples of polymeric insulation materials, cable Indenter measurement became traditional.

The method based on measurement of the recovered voltage is successfully applied to assessment of a power cable condition. For the power cables with the impregnated paper insulation the condition estimation rules have been developed.

\section{DIFFERENTIAL SCANNING CALORIMETRY FOR MONITORING AND PREDICTION OF A CABLE CONDITION WITH POLYOLEFIN INSULATION}

Most widely in systems of in-reactor control the in-containment I\&C cables with polyolefin insulation materials are applied, in particular, with XLPE insulation.
Ageing of these cables is defined as their oxidation under the influence of the elevated temperature and radiation as well as the direct destruction of molecular chains by an ionizing radiation. A process of polyethylene insulation ageing has two stages: a period of the antioxidant consumption to critical value (the induction time) and chain oxidation time. The time of chain oxidation is defined by environment temperature; at temperature about $60^{\circ} \mathrm{C}$ it makes no more than one year [3]. Hence, in hot spots of operation a service life of cables with polyethylene insulation will correspond to the induction time.

To prevent oxidation the antioxidants are entered into the insulation of cables. The conservative estimate of polyolefin insulation material life is realized on the basis of two tests of microsamples in a cell of the DSC device: isothermal mode for measuring oxidation time (OIT) and mode of temperature ramp for measuring oxidation temperature (OITP). The values of OIT and OITP are directly proportional to concentration of an antioxidant. It means testing of several milligrams of polyolefin insulation for thermal resistance in a device cell at constant temperature in oxygen atmosphere, or at constant rate of temperature rising prior to the beginning of chain oxidation reaction, which is recorded on occurrence of an exothermic peak on a thermogram. By the results of such tests of insulation microsamples, an expected life of cables can be calculated from the equations (1) and (2) taking into consideration that the conditions of cable operation are remained invariable. The cable expected life by OIT value, $\tau_{E L_{-} O I T}$, is calculated from the equation [1]:

$$
\tau_{E L_{-} \text {OIT }}=\tau_{\text {test }} \cdot \frac{O I T_{\text {test }}-O I T_{\text {lim }}}{O I T_{\text {ini }}-O I T_{\text {test }}}
$$


where $\tau_{\text {test }}$ and $O I T_{\text {test }}$ - the time of cable operation and OIT value at the moment of the condition monitoring, $O I T_{i n i}$ and $O I T_{\lim }$ - the initial and limiting values of OIT. Values of $O I T_{i n i}$ and $O I T_{\text {lim }}$ are predetermined under laboratory conditions for each kind of insulation. Eq.(1) is simply derived when assuming direct proportional dependence $\tau_{\text {OIT }}$ on OIT change. Such assumption provide conservative estimation of cable expected life.

The cable expected life by OITP value is calculated from the equation [1]:

$$
\tau_{E L_{-} \text {OITP }}=\tau_{\text {test }} \cdot \frac{O I T P_{\text {test }}-O I T P_{\text {lim }}}{O I T P_{\text {ini }}-O I T P_{\text {test }}} \cdot \frac{O I T P_{\text {ini }}+273}{O I T P_{\text {lim }}+273},
$$

where OITP $_{\text {test }}$, OITP $_{\text {ini }}$, OITP $_{\text {lim }}-$ controllable, initial and limiting values of OITP. Eq.(2) has been received on the basis of experimentally verified dependence of 1/OITP on an aging time [4].

A disadvantage of such approach is the necessity of preliminary determination of initial and limiting values of OIT or OITP for each type of the polyolefin insulation that is connected with a great volume of preliminary works, including on artificial ageing of polyolefin insulation.

If boundary data on OIT and OITP are unavailable, the expected life $\tau(T)$, of polyolefin insulation for thermal ageing under operation represents an oxidation time and can be calculated from the equation $[4,5]$ :

$$
\tau_{E L_{-} T}(T)=a \cdot \exp \left(\frac{E_{a}}{R T}\right),
$$

where $E_{a}$ - the activation energy of antioxidant oxidation process; $a$ - the constant preexponential multiplier; $R$ the Boltzmann constant; $T$ - the operation temperature. For determination of values $E_{a} \& a$, measurements of both OIT and OITP are carried out.

For determination of $E_{a}$, tests of insulation microsamples in the device DSC cell are carried out at various temperatures of isothermal endurance $T_{\text {iso }}$ in a range from 200 to $230^{\circ} \mathrm{C}$. By results of tests proceeding from linear dependence $\ln (O I T)$ on $1 / k T_{\text {iso }}$, values of activation energy are defined (see Fig. 1).

To define the value, $a$, measurements of microsamples OITP are carried out at different velocities of temperature rising $\alpha$. Usually the velocity, $\alpha$, is selected in a range from $2 \mathrm{deg} / \mathrm{min}$ to $10 \mathrm{deg} / \mathrm{min}$ in the DSC device cell; the initial temperature of measurements, $T_{0}$, corresponds to room one. Then value $a$ is calculated from the equation [4]:

$$
a=\frac{1}{\alpha} \int_{T_{0}}^{O I T P} e^{-\frac{E_{a}}{k T}} d T
$$

For estimation of radiation influence on service life of cables, the accelerated thermoradiation tests of widely used cable samples in internal control systems of WWER-1000 of KPETIng type with the XLPE insulation were conducted. The irradiation was carried out at a temperature of $75^{\circ} \mathrm{C}$ and the absorbed dose rate of $0,6 \mathrm{~Gy} / \mathrm{s}$. From the results of these tests (see Fig.1) it has been established that the $E_{a}$ of XLPE insulation of the KPETIng cable does not vary up to the absorbed dose of $1590 \mathrm{kGy}$ and the value $a$ exponentionally depends on a dose, $D$. In other words, it follows from the data in Fig. 1 that in this case radiation exposure causes a shift of $\ln (O I T)$ dependence on $1 / k T_{\text {iso }}$ down in an axis of ordinates owing to decrease in a value of $a$ in Eq.(3). For our estimates, let us assume that this shift is described by the following dependence:

$$
\ln \left[\tau_{O I T}(T, D=0)\right]-\ln \left[\tau_{O I T}(T, D=1590 k G y)\right]=k \cdot D,(5)
$$

where $\tau_{O I T}$ - is the oxidation time of an insulation sample in a cell of the DSC device.

For conservative estimates of radiation exposure on the expected life of XLPE insulation let us assume that the dependence described by Eq.(5) is valid not only for insulation microsamples under testing in the cell of the DSC device, but still at lower operating temperatures for a cable insulation.

In view of Eq.(3) and (5), for the expected life estimation of cables with XLPE insulation under conditions of simultaneous exposure to $\gamma$-radiation and elevated temperature, it is possible to use the equation:

$$
\tau_{E L_{-} T R}(T, \gamma)=a \cdot \exp \left(\frac{E_{a}}{R T}\right) \cdot \exp (-k \cdot D),
$$

where $k$ - is the factor depending on a type of insulation.

From the results of the accelerated tests and using Eq.(3) and Eq.(6), the expected life assessment of the KPETIng cables was conducted at the elevated temperature and at simultaneous influence of $\gamma$-radiation and elevated temperature that is shown in Fig. 2. When carrying out the calculations, it was accepted that the dose rate is corresponds to a maximum possible design one for these cables. These estimations show that the cable service life in the in-reactor control systems makes not less than 60 years at the maximum design levels of degradation factors without taking into consideration the influence of damaging factors of design basis events.

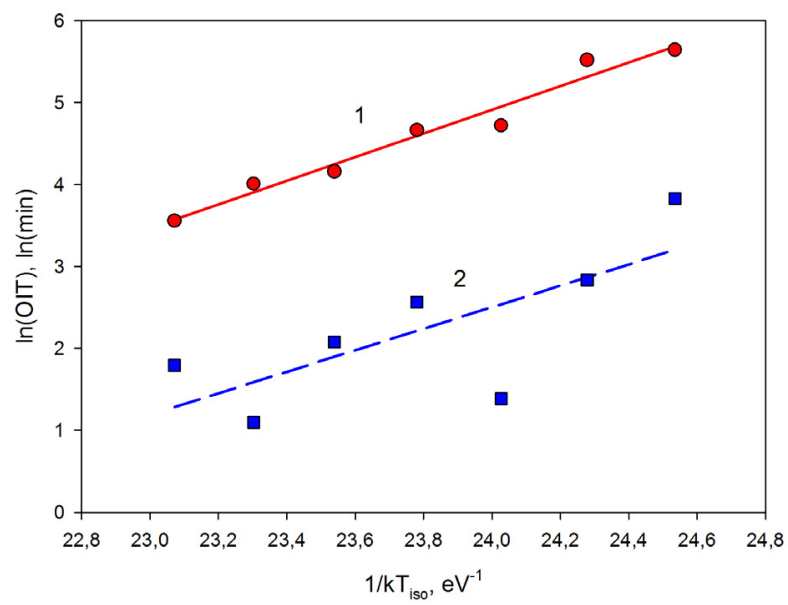

Fig. 1. Dependence of induction time on temperature in a cell of DSC device for insulation of KPETIng cable samples: initial (1) and after the thermoradiation ageing (2) at the absorbed dose of $1590 \mathrm{kGy}$ and temperature of $75^{\circ} \mathrm{C}$ 


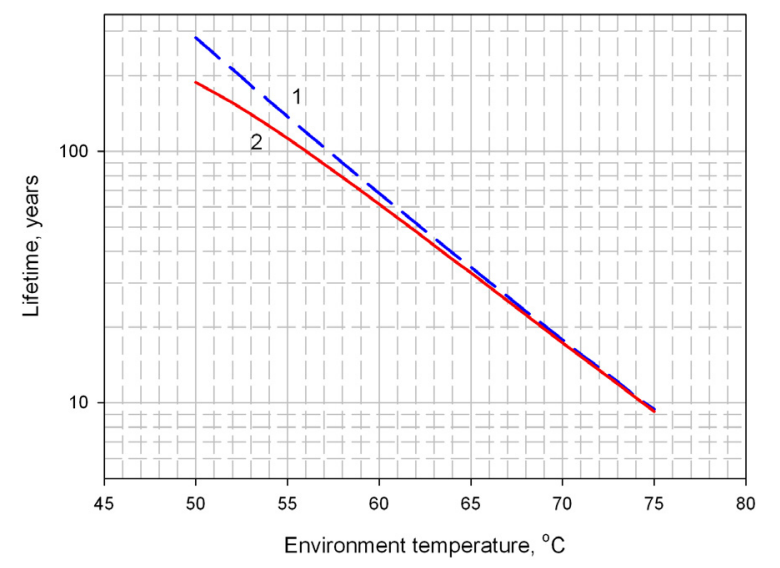

Fig.2. The design service life of KPETIng cable insulation at thermal (1) and thermoradiation (2) ageing at the maximum possible designed dose rate for WWER-1000

As well as any monitoring method, DSC of microsamples it has its restrictions. They are obvious for the DSC method: if ageing of polyolefin insulation goes without the antioxidant consumption, the given method is not applicable. Such situation in our practice really took place at one of the nuclear power plant units when assessing the service life of suspension consoles of ionization chambers, which was defined by a condition of the cable with the polyethylene insulation and jacket, laid in the suspension console. An environment for this cable was nitrogen; therefore the ageing of insulation under the radiation exposure proceeded by means of direct destruction of polymer molecular chains. In this case the service life estimation of the cable to be carried out by a value of relative elongation at break; for this purpose one suspension console has been withdrawn from operation.

At the same time, it is qualitatively clear that under usual operation conditions a change in mechanical and electric properties of polyolefin insulation is possible only at the second stage of ageing, i.e. after when the antioxidant content reaches a critical value. It means that it is possible to fit such guaranteed overestimated limiting value of $O_{\text {ITP }}$, at which influence of damaging factors of design accidents after ageing in normal operation conditions will not lead to development of polyolefin chain oxidation process. In other words, it is possible to estimate cable service life taking into account the influence of design accidents factors on the cable by a OITP value without carrying out of expensive tests simulating damages during design accidents. A conservatism degree of such approach will be defined by $O I T P_{\text {lim }}$ value choice.

\section{INFRARED SPECTROSCOPY FOR THE MONITORING AND DIAGNOSTICS OF CABLES WITH POLYMERIC INSULATION}

Application of the IR Fourier spectroscopy with an attachment of the broken total internal reflection for work with microsamples gives possibility to define a type of polymeric insulation and to estimate a degree of its ageing on characteristic band of absorption spectra.
Now the obtained experimental data have allowed developing a technique for assessment of service life of the most widely used out of-containment PVC insulation cables jacket with various types of plasticizers. At that, the technique takes into account that the ageing can proceed by means of both plasticizer desorption and destruction of polymer molecular chains.

The thermal ageing of PVC plastics under NPP operating conditions is defined by plasticizer desorption. The accepted in Russia plasticizers represent complicated ethers of carboxylic acids that gives an opportunity to determine a concentration of the plasticizer in a sample by optical density of a peak at a wave length of $1720 \mathrm{~cm}^{-1}$, i.e. by the band intensity of carbonyl valent fluctuations (Fig. 3), or by a band intensity of its first overtone of $3450 \mathrm{~cm}^{-1}$.

Except plasticizers on the basis of complicated ethers of carboxylic acids, plasticizers on the basis of phosphates is added to the most widespread commercial PVC plastics for jackets, to which the $1190 \mathrm{~cm}^{-1}$ characteristic absorption band corresponds. By the intensity of this band it is possible to control quantitatively their concentration taking into consideration that its initial concentration makes about $6 \%$ in commercial insulation.

Taking into consideration the exponential dependence of plasticizer concentration on time of ageing, the expected life by the given indicator is determined by the equation:

$$
\tau_{E L_{-} C}=\tau_{\text {test }} \cdot \frac{\ln C_{i n i}-\ln C_{\text {lim }}}{\ln C_{i n i}-\ln C_{\text {test }}},
$$

where $C_{i n i}, C_{\text {lim }}, C_{\text {test }}$ are the initial, limiting content and measured values of plasticizer, respectively.

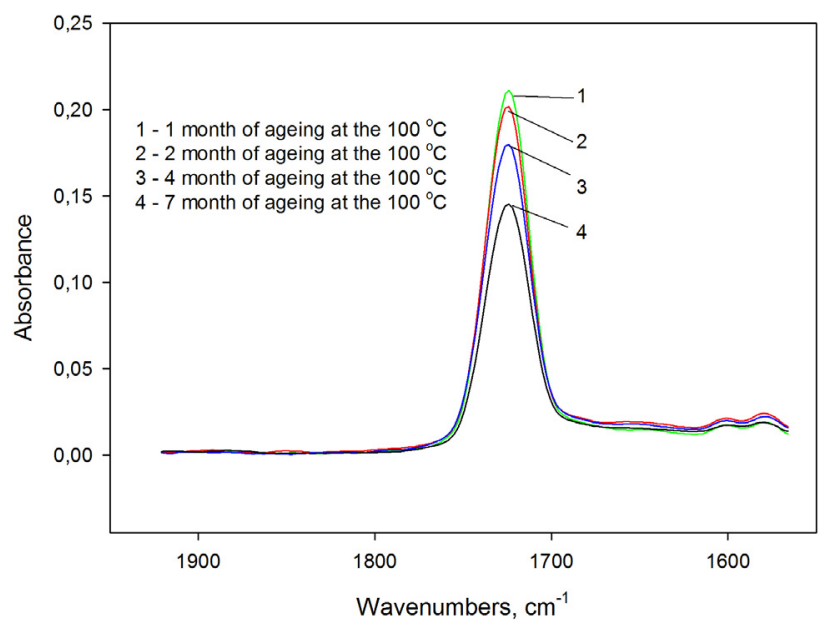

Fig.3. IR spectra of the jacket's PVC plastics for various levels of artificial thermal ageing

The second mechanism of ageing of PVC plastics caused by destruction of molecular chains can take place under radiation exposure or at the elevated humidity, therefore for today in practice in nuclear power plant premises it meets seldom. The ageing assessment by the given mechanism of ageing is carried out by the characteristic change in IR of spectra in the region of $2000-3700 \mathrm{~cm}^{-1}$ because of destruction of polymeric chains (see Fig. 4). 


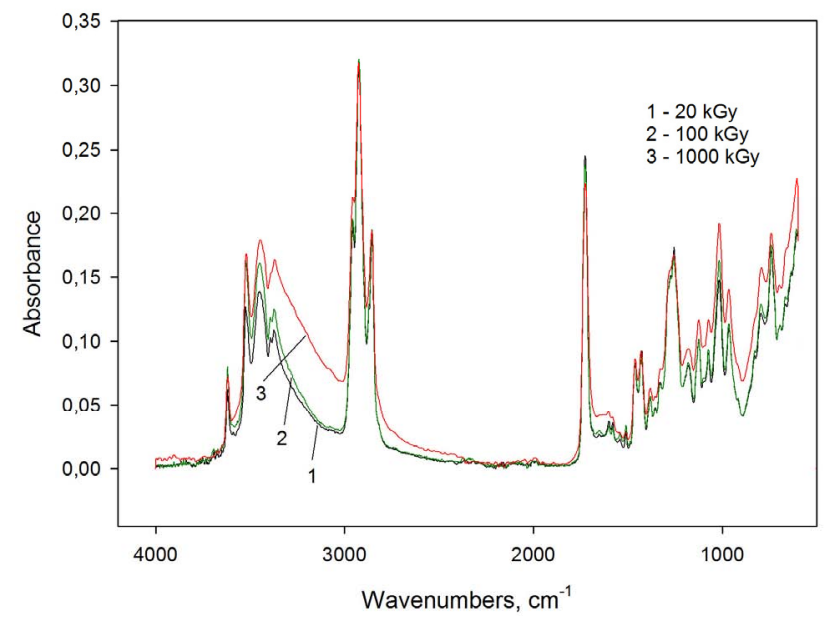

Fig.4. IR spectra of jacket PVC plastics for various doses of gamma-irradiation

The background absorption starts to grow that is caused by occurrence of secondary bond and free $\mathrm{OH}$-groups due to destruction of polymeric chains, including the plasticizer destruction. For the quantitative description of the integrated absorption of hydroxyl in this area, the indicator $D$ is used which is defined as ratio logarithm of typical IR-spectrum absorption bands product [6]:

$$
D=500 \cdot \lg \left(\frac{I_{2200} \cdot I_{3700}}{I_{2700} \cdot I_{3050}}\right),
$$

where $I_{2200}, I_{2700}, I_{3700}, I_{2200}$ - are the experimentally determined values of transmission in reference points. For definition of the cable expected life by the $D$ indicator, the following equation is used:

$$
\tau_{E L_{-} D}=\tau_{\text {test }} \cdot \frac{D_{\text {lim }}-D_{\text {test }}}{D_{\text {test }}-D_{\text {ini }}},
$$

where $D_{\text {lim }}, D_{\text {ini }}, D_{\text {test }}$ - are limiting, initial and measured values of integrated absorption, respectively.

The destruction degree of polymeric chains can be also controlled by the $1650 \mathrm{~cm}^{-1}$ absorption band corresponding to the $\mathrm{C}=\mathrm{C}$ double bond.

Thus, the use of IR spectroscopy allows effectively monitoring two basic processes of PVC plastics ageing: plasticizers desorption and destruction of polymeric chains. The least value obtained in calculations by Eq.(7) and Eq.(9) is accepted as the expected life of a cable from PVC insulation materials.

It is also possible to define a composition of PVC plastics by IR spectra. In particular, by characteristic bands of absorption in the range of $3000-3600 \mathrm{~cm}^{-1}$, for example, it is possible to learn whether the PVC plastics contain the additives, which limit intensity of burning.

Application of the IR spectroscopy for condition monitoring of polymeric electric insulation is very useful, including, for identification of a kind of the applied polymeric material as well as for specifying a mechanism of cable ageing. For example, it was revealed that under operation at temperatures of about $60{ }^{\circ} \mathrm{C}$ the ageing of polyethylene insulation of a KPOBov- type cable goes more intensively than the ageing of its internal polyethylene jacket.
Results of the IR-Fourier spectroscopy applied to the artificially aged samples of the given type of a cable at $100{ }^{\circ} \mathrm{C}$ have confirmed the presence of intensive oxidation of polyethylene insulation at the actual absence of the polyethylene internal jacket oxidation. In Fig. 5, the dependences of carbonyl groups accumulation in insulation

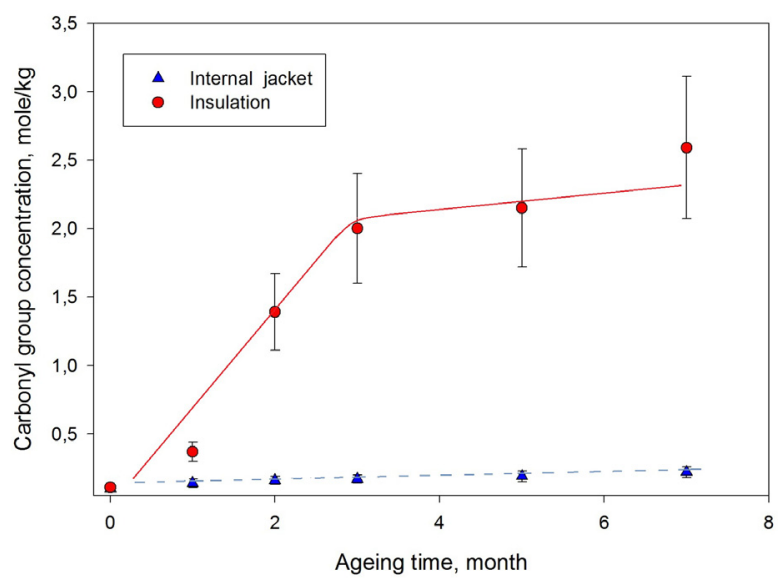

Fig.5. Accumulation of carbonyl groups in insulation, $(\bullet)$, and in internal jacket, $(\boldsymbol{\Delta})$, of KPOBov cable during thermal aging at $100{ }^{\circ} \mathrm{C}$ temperature

and in an internal jacket, which were defined by intensity of the band absorption at a wavelength of $1720 \mathrm{~cm}^{-1}$ are presented. It is obvious that in three months the insulation oxidation reaches its limiting value. The only reason of the intensive insulation ageing is the catalytic influence of conductor's copper ions on processes of thermal oxidation of polyethylene as in the cable insulation of the given type made about 20 years ago copper deactivators were absent.

\section{ASSESSMENT OF A CABLE CONDITION BY CABLE INDENTER MEASUREMENTS}

Cable polymeric Indenter is traditionally applied to estimating a degree of I\&C cable ageing. The essence of the given approach consists in definition of cable jacket rigidity at its deformation in a local point by a special probe. In the cable Indenter of an IPAM-2 model the jacket deformation is made by the probe, which moves with a constant velocity. The condition assessment is carried out by a compression module, $M$.

It is should note the limitation of using the given method by types of the tested insulation materials as well as by ageing mechanisms. It is effective for estimation of ageing which is caused by destruction of polymer molecular chains or for PVC insulation at plasticizer desorption. The method is not applicable to the cross-linked materials, for example to XLPE, or for polymers, at ageing of which the linkage processes of molecular chains with formation of a spatial grid are dominated. Now the condition of cable PVC jackets and jackets made of some types of cable rubbers is monitored by measurement of the compression module. For the latter, it is the single method for nondestructive condition monitoring.

A dependence of the compression module, $M$, on the temperature, $T$, of the monitored sample has been established and it is described by the following empiric equation: 


$$
M(20)=M(T)-A \cdot T \cdot \exp \{-B[1000 /(T+273)-2,77]\}
$$

where $M(20), M(T)$ are the compression modules at temperature of $20{ }^{\circ} \mathrm{C}$ and measurement temperature, respectively, and $A$ and $B$ - the constants characteristic for the given type of insulation. For PVC jackets, a dependence of the compression module on temperature should be taken into account when carrying out measurements that is obvious from the experimental data presented in Fig.6.

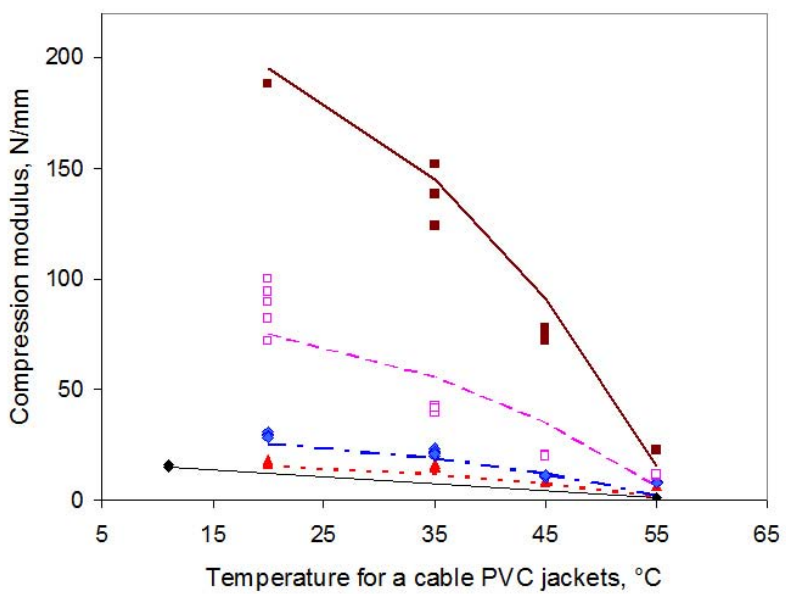

Fig.6. Influence of temperature on the value of the compression module for the PVC cable jacket with a different level of ageing

For cables of NGRSHM and KGN types with rubber jackets, the correlation dependence between relative elongation at break and the compression module has been experimentally received (see Fig. 7).

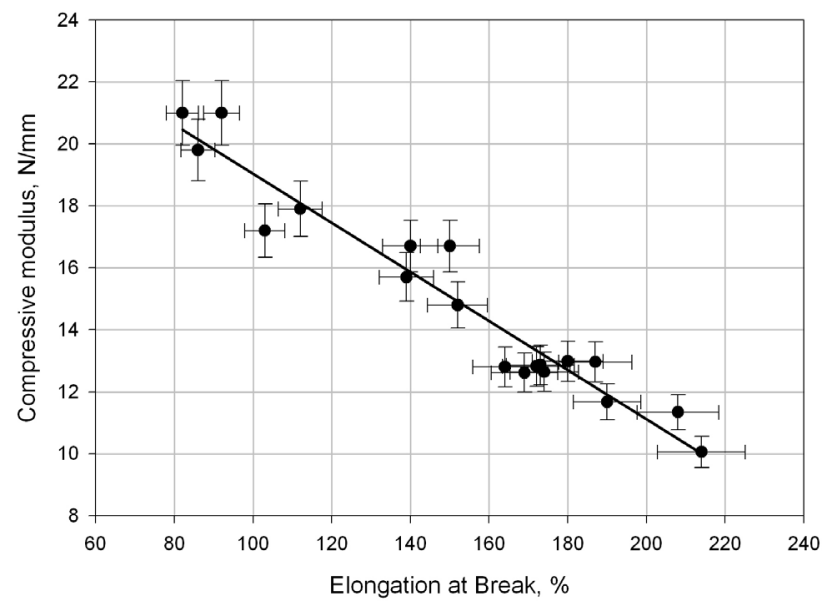

Fig.7. Correlation relationship between the compression module and elongation at break for cable rubbers

For the expected life estimation by the compression module the following equation is used:

$$
\tau_{E L_{-} M}=\tau_{\text {test }} \cdot \frac{M_{\text {lim }}-M_{\text {test }}}{M_{\text {test }}-M_{\text {ini }}},
$$

where $M_{\text {ini }}, M_{\lim }, M_{\text {test }}$ are the initial, limiting, and measured values of the compression module, respectively.

\section{APPLICATION OF THE RECOVERED VOLTAGE FOR ESTIMATION OF POWER CABLE CONDITION}

The recovered voltage, $\mathrm{RV}$, is a voltage on the disconnected isolator measured after its charging with the voltage, $U_{c h}$, chosen beforehand during the time, $t_{c h}$, and its subsequent partial preliminary discharging during the time, $t_{d}$, chosen beforehand. The RV measurement, by its physical nature, is the most sensitive electric method for control of defects formation in electric insulation as two basic processes define its value: volume-charging polarization and electric conductivity.

At the same time, the wide implementation of this method in practice is restrained due to unavailability of full understanding how to receive quantity indicators from the RV curve for the description of insulation ageing and humidifying as till now an attempt to allocate the contribution of electric conductivity and polarization in this curve was not a success. The problem becomes complicated still that the RV value and form depend on both temperature and geometry of the monitored insulation, insulation type, and from the parameters directly forming RV value and form: $U_{c h}, t_{c h}$, $t_{d}$.

We have offered a new approach to estimation of the electric insulation condition on the basis of RV analysis with the aim to control a condition of the power cables with the impregnated paper insulation, which were laid out of containment. The nuclear power plant's personnel spares them the greatest attention during preventive repairs because of ageing of terminal sealing-off and insulation drying out on vertical sections of cable lines after long-term operation at high environment temperature and vibration from the working equipment.

For the description of the RV curve, $U_{r}(t)$, a semiempirical model was used in the form of the exponent sum with parameters, $A_{i}$ and $\tau_{i}$ :

$$
U_{r}(t)=\sum_{i=1}^{n} A_{i} \cdot \exp \left(-\frac{t}{\tau_{i}}\right),
$$

where $t$ is the time. At that, for description of the really measured curves of RV in a time range of $0,1-2000 \mathrm{~s}$, it is enough to take $n$ value equal to 4 or 5 . The typical curve, $U_{r}(t)$, and its components defined by model (11) are presented on Fig. 8. Here, as it is traditionally accepted, a value of $\mathrm{RV}$ is negative; therefore short-lived components have a positive value.

Unfortunately, $A_{i}$ and $\tau_{i}$ as well as a maximum of the recovered voltage, $U_{r m}$, cannot be used as universal indicators of the electric insulation condition as they depend on geometry (length and section) of the tested cables and parameters of preliminary charging. Viewing the semiempirical model (11), we noted the following aspects:

- relaxation processes with the characteristic times $(0,2-$ 50) $t_{d}$ are responsible for initial grow of $U_{r}(t), U_{r m}$ is formed at the expense of relaxation processes with the times of $100 \cdot t_{d}$-order and bulk electric conductivity of insulation material [7];

- the sum of intensities of short-lived positive components, $A_{+}$, is directly proportional to polarization of tested electric insulation at the $t=0$ moment, i.e. at the 
moment of the measurement beginning. Really, before the moment of measurements the conductor and metal shield are locked for $t_{d}$ time, depolarization velocity of insulation spatial charges at this time is defined by a value of the metal conductor conductivity, i.e. it is practically constant for all cables;

- $\quad$ the square, $S_{+}$, under short-lived positive components in "voltage - time" coordinates (see Fig. 8) is proportional to the bulk charge caused by relaxation of short-lived positive components.

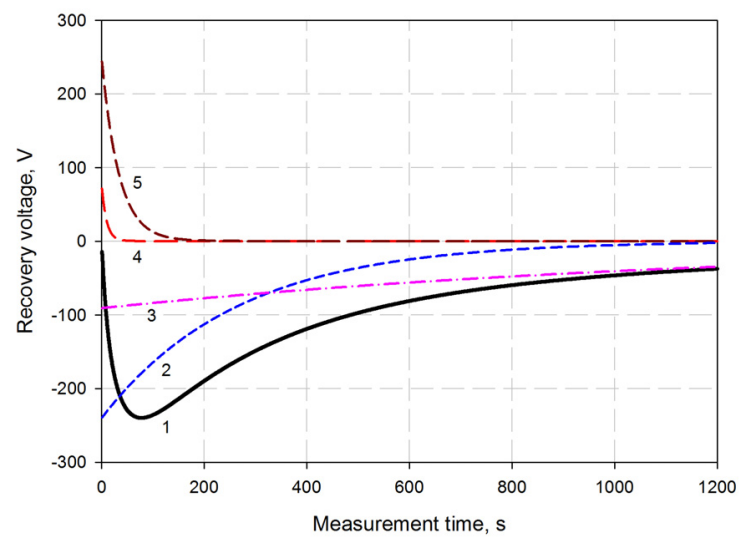

Fig.8. Typical curve of the recovered voltage (1) and its components $(2-5)$

Based on these aspects, two indicators of the insulation condition [8] have been introduced. The first is the $R U 1_{m}$ indicator, which is directly proportional to the bulk polarization (a volume charge) of insulation and defined as:

$$
R U 1_{m}=10 \cdot\left|\frac{U_{r m}}{A_{+}}\right| .
$$

The decrease in the $R U 1_{m}$ value for the impregnated-paper insulation can be caused by such typical defect as paper drying out.

The second is the $R U 2_{m}$ indicator directly proportional to bulk electric conductivity of insulation and defined as:

$$
R U 2_{m}=100 \cdot\left|\frac{U_{r m}}{S_{+}}\right| .
$$

The increase in this indicator can be caused by insulation humidifying. (The module sign and factors in equations are entered here for convenience).

Fig. 9 shows $U_{r}(t)$ and $U_{r}(t) / A_{+}$, and $U_{r}(t) / S_{+}$for five cables of AASHv 3x150 type with impregnated-paper insulation under different level of ageing and humidifying. For the 1st cable of $540 \mathrm{~m}$ in length, the insulation is unaged, the integrated humidifying of insulation makes $0,7 \%$, a polarization index is equal to 4,4 (a humidifying degree was defined by the method of frequency dielectric spectroscopy from a minimum value of a dielectric loss tangent, $\operatorname{tg} \delta_{\min }$ [9], a polarization index was defined as a relation of the insulation resistance at 10 minutes to the resistance at 1 minute). The insulation ageing of the 2nd and 4th cables of 36 and $33 \mathrm{~m}$ in length can be characterized as average; an index of polarization for them is equal to 3,3 and 2,0; humidifying has made $1,4 \%$ and $0,4 \%$, respectively. I.e. the 2 nd cable ageing, to the great extent, is caused by insulation humidifying, and the 4th one - by the paper destruction. The 5th cable insulation of $80 \mathrm{~m}$ in length is strongly humidified in a local place; its integrated humidifying is equal to $2 \%$ and a polarization index - 1,1. Paper insulation of the 3rd cable of $80 \mathrm{~m}$ in length has been completely dried out and has turned out fragile (the cable has been removed from operation).
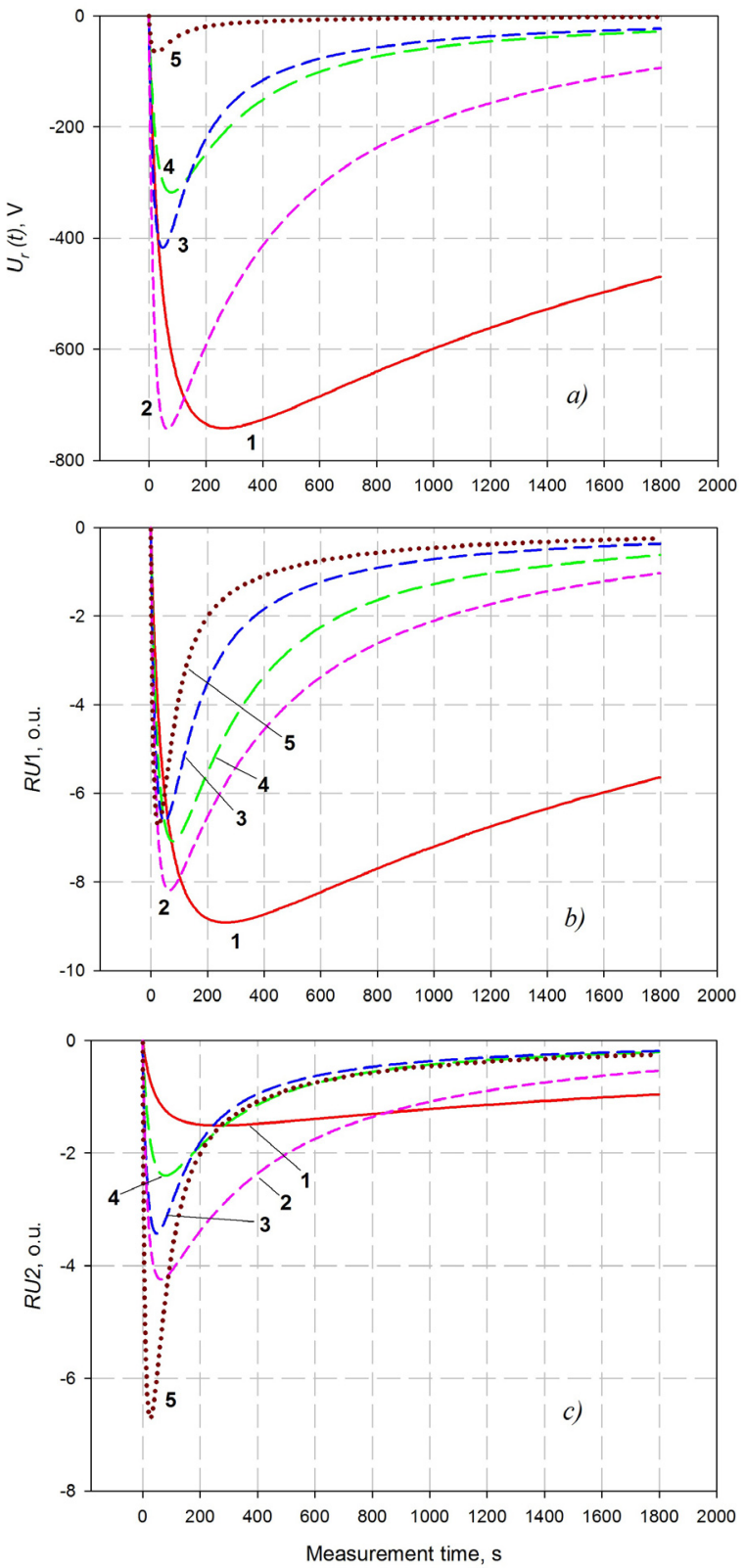

Fig. 9. Curves of RV for representative cables with impregnated-paper insulation $(a)$ and their normalized values for estimating a polarization degree $(b)$ and electric conductivity $(c)$

From the results of the condition monitoring of more than 300 cables with impregnated-paper insulation, rules for assessment of their condition by the method of the recovered voltage have been developed, which are presented in Table 1. With this aim, correlation dependences between $R U 1_{\mathrm{m}}, R U 2_{\mathrm{m}}$ and the traditional indicators of an insulation condition were analyzed. Fig. 10 shows an example of such dependence between $R U 2_{\mathrm{m}}$ and moisture content in insulation. 


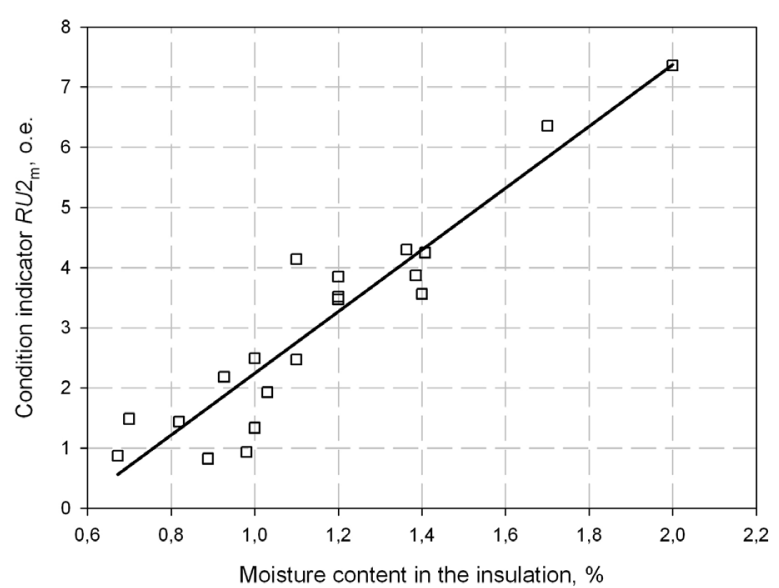

Fig. 10. Correlation dependence between the $R U 2_{\mathrm{m}}$ indicator and the moisture content for the impregnated-paper insulation

Table 1. A degree of paper insulation ageing and a cable condition by $R U 1_{\mathrm{m}}$ and $R U 2_{\mathrm{m}}$ indicators

\begin{tabular}{|c|c|c|}
\hline $\begin{array}{c}\text { Cable condition/ } \\
\text { Degree of insulation } \\
\text { ageing }\end{array}$ & $\begin{array}{c}\text { Indicator } \\
R U 1_{\mathrm{m}}\end{array}$ & $\begin{array}{c}\text { Indicator } \\
R U 2_{\mathrm{m}}\end{array}$ \\
\hline \hline $\begin{array}{c}\text { Limiting / } \\
\text { Limiting degree of } \\
\text { insulation drying } \\
\text { out }\end{array}$ & \multicolumn{2}{|c|}{$R U 1_{\mathrm{m}}<6,6$} \\
and $R U 2_{\mathrm{m}} \leq 0,85$
\end{tabular}

\section{CONCLUSIONS}

The presented nondestructive condition monitoring methods for I\&C cables give the opportunity to make a periodic estimation of their expected life without destruction of cable lines.

The new approach to the estimation of a technical condition of power cable electric insulation on the basis of the recovered voltage measurement allows monitoring a change in a bulk charge condition and electrical conductivity of insulation by results of one measurement.

For power cables with the impregnated paper insulation, which are laid out of containment in old power units of the nuclear power plants, this approach enables to monitor such typical defects under operation as drying out of the impregnant and insulation humidifying across all the way of its ageing from faultless to limiting conditions.

\section{REFERENCES}

[1] IAEA-TECDOC-1188. Assessment and management of ageing of major nuclear power plant components important to safety: In-containment instrumentation and control cables, Volume 1-2, ISSN 1011-4289. - Vienna: IAEA, 2000.

[2] Kononenko A.I., Tsikanin A.G., et al., "Improvement of techniques for nondestructive diagnostics of a cables condition and management of their service life in power units of nuclear power plant”. Plenary and section reports of the 4th International Scientific and Technical Conference "Safety, Efficiency and Atomic Engineering Economy", C.207 - 214., Moscow, VNIIAES, 16-17 June, 2004.

[3] Kononenko A.I., et al., "Prediction of an induction period for polyethylene cable insulation”, Issues of Nuclear Science and Engineering. Series: Physics of Radiation Effect on Radio-electronic Equipment. Issue Nos.1-2, S.31-40, Moscow, 1995.

[4] Shlyapnikov Yu.A., Kiryushin S.K., Mariin A.P. Antioxidation stabilization of polymers. "Chemistry", Moscow, 1986, 256 p.

[5] Mason L.R., "Reynolds A.B. "Comparison of oxidation induction time measurements with values derived from oxidation induction temperature measurements for EPDM and XLPE polymers”. Polymer Engineering and Science, July, 1998, vol. 38, No.7.

[6] Bochkarev R.N.. Filatov N.S., Environmental Aging of Polyvinylchloride-based materials. - Novosibirsk: Science. Siberia branch, 1990г. - 118 р.

[7] Kononenko A. I. et al., "Recovered voltage phenomenon and prospects for its use for assessment of a polymer condition”. Polymer Science, Ser. A, 2006, Vol. 48, No. 11, pp. 1190-1195.

[8] Kononenko A.I., Hohryakov A.V., "Estimation of a paper electric insulation condition by the results of measurements of the recovered voltage", Electrical Engineering, S.47-55, No.5, 2010.

[9] Neimanis R., et al., "Determination of moisture content in mass impregnated cable insulation using low frequency dielectric spectroscopy". IEEE Power Engineering Society Summer Meeting, Seattle, USA, July 16-20, 2000. 